RAL-93-071

October 1993

\title{
Determining the gluon distributions in the proton and photon from two-jet production at HERA
}

\author{
J.R. Forshaw and R.G. Roberts \\ Rutherford Appleton Laboratory, \\ Chilton, Didcot OX11 0QX, England.
}

\begin{abstract}
Two-jet production from the direct photon contribution at HERA is a sensitive measure of the small- $x$ gluon in the proton. We propose measurements of ratios of the jet cross-sections which will clearly distinguish between gluons with or without singular behaviour at small $x$. Furthermore, we show that analogous ratio measurements for the resolved photon contribution provide a sensitive way of determining the gluon distribution in the photon.
\end{abstract}




\section{Introduction}

One of the aims of HERA is to study the structure of the proton and in particular to learn about the distribution of gluons at small $x$ (i.e. $x \lesssim 10^{-2}$ ). Here the gluon is expected to be dominant and we are in an unexplored region of lepton-hadron scattering. The latest measurements of the proton structure function $F_{2}^{p}\left(x, Q^{2}\right)$ from H1 [1] and ZEUS [2] show the distinct rise for $x \lesssim 10^{-2}$ which has encouraged speculation that this may be a signal of socalled 'Lipatov' behaviour [3]. On the other hand it is possible to generate such a rise using conventional leading $\log Q^{2}$ evolution from a 'valencelike' gluon distribution at $Q_{0}^{2}=0.3 \mathrm{GeV}^{2}$ 四. Measuring $F_{2}^{p}$ however is only an indirect probe of the gluon and this is the main reason why the gluon is the least constrained of all the parton distributions to date. There have been several methods proposed which aim to extract the small- $x$ gluon content of the proton at HERA rather more directly [5, 6, 7].

Here, we present an analysis of two-jet photoproduction which utilises the separation of events into so-called 'direct' and 'resolved' components. Through the construction of appropriate cross section ratios we show that HERA should provide a sensitive and direct measurement of the small- $x$ gluon in the proton in addition to revealing important information regarding the gluon in the photon.

The first step we take is analogous to the procedure previously considered for hadronic two-jet production [8, 9] where the configuration of 'same-side' jets allows very small $x$ values of the initial partons to be examined. At HERA we propose isolating the contribution to jet photoproduction from 'direct' photon interactions (see fig.1(a)). We focus on the region where both jets have equal rapidities and are travelling in the electron direction (which we define to be that of positive rapidity). Such large and positive rapidities mean that we are sensitive to the small- $x$ gluon within the proton.

The second step is to consider contributions like those of fig.1(b), where the photon is resolved into its partonic components. We now study the region of negative jet rapidities where, conversely to the first step, the cross section is sensitive to the parton distributions in the photon for values of $x_{\gamma}$ around $0.2-0.5$. With the proton gluon distribution already constrained from step one, the resolved photon contribution to two-jet production provides a sensitive measure of the parton distributions in the photon. The possibility of using the two-jet data from HERA to provide information on the parton content of the photon, especially on the 
gluon distribution has been emphasised by several groups [10, 11, 12]. We wish to emphasise that measurement of ratios of the two-jet cross sections is a particularly clean and sensitive way of discriminating between different parametrisations of the gluon distribution which tends to minimise the experimental and theoretical uncertainties involved.

\section{Step 1 - the gluon in the proton}

To begin with, consider the direct photon contribution to two-jet production, e.g. see fig.1(a). The two-jet cross section may be written to leading order (LO) as

$$
\frac{d^{3} \sigma^{d i r}}{d y_{1} d y_{2} d p_{T}^{2}}=z f_{\gamma / e}(z) x f_{j / p}(x, \mu) \frac{d \hat{\sigma}(\gamma j \rightarrow k l)}{d \hat{t}}
$$

where $f_{\gamma / e}(z)$ is the probability that the incoming electron will emit an effectively real photon with a fraction $z$ of its momentum and $f_{j / p}(x, \mu)$ is the momentum distribution of parton $j$ inside the proton evaluated at scale $\mu$. The laboratory rapidities $\left(y_{1}\right.$ and $\left.y_{2}\right)$ of the final state partons (emitted with transverse momentum $p_{T}$ ) can be expressed in terms of the momentum fractions $z$ and $x$ :

$$
\begin{gathered}
z=\frac{p_{T}}{\sqrt{s}} \sqrt{\frac{E_{p}}{E_{e}}}\left(e^{y_{1}}+e^{y_{2}}\right) \\
x=\frac{p_{T}}{\sqrt{s}} \sqrt{\frac{E_{e}}{E_{p}}}\left(e^{-y_{1}}+e^{-y_{2}}\right) .
\end{gathered}
$$

The energies of the incoming electron and proton are $E_{e}$ and $E_{p}$ respectively and the centre-ofmass energy is $\sqrt{s}=2 \sqrt{E_{e} E_{p}}$. In this analysis we shall study only the configuration where the two jets have equal rapidities, i.e. $y_{1}=y_{2}=y$. At HERA energies, choosing $y \sim 1$ and $p_{T}=5$ $\mathrm{GeV}$, we find $x \simeq .003$.

There are two subprocesses in the direct photon contribution: (i) $\gamma g \rightarrow q \bar{q}$ and (ii) $\gamma q(\bar{q}) \rightarrow g q(\bar{q})$ and the LO cross section of eqn.(1) takes the simple form

$$
\left.\frac{d^{3} \sigma^{d i r}}{d y_{1} d y_{2} d p_{T}^{2}}\right|_{y_{1}=y_{2}=y}=\frac{\pi \alpha \alpha_{s}}{4 p_{T}^{4}} z f_{\gamma / e}(z)\left[\left(\sum_{i} e_{i}^{2}\right) x g(x, \mu)+(10 / 3) F_{2}^{p}(x, \mu)\right] .
$$

Since $x g(x, \mu)$ is much larger than $x \bar{q}(x, \mu)$ at small $x$ then (i) dominates the cross section for $x \lesssim 10^{-2}$. Thus evaluating ratios of the above cross section at different values of $y$ effectively measures the ratio of the gluon distribution at different values of $x$. We suggest measuring the ratio $\sigma_{d i r}(y) / \sigma_{d i r}(y=-0.5)$ (where we have integrated over all $p_{T} \geq 5 \mathrm{GeV}$ in eqn.(3) to 
construct $\left.\sigma_{\text {dir }}(y)\right)$. Fig.2 shows the expectations for this ratio from various proposed gluons: $M R S D 0^{\prime}, M R S D-^{\prime}\left[13\right.$ and $G R V$ [ [T] The $D-^{\prime}$ curve is generated from a singular gluon which, at $\mu^{2}=4 \mathrm{GeV}^{2}$ and small $x$, behaves as $x g(x, \mu) \sim x^{-1 / 2}$ which is in contrast to the $D 0^{\prime}$ curve which corresponds to $x g(x, \mu) \rightarrow$ constant as $x \rightarrow 0$ at $\mu^{2}=4 \mathrm{GeV}^{2}$. The $G R V$ prediction leads to a singular gluon distribution for $\mu^{2} \gtrsim 1 \mathrm{GeV}^{2}$ due to the long evolution time which results from starting evolution at low scales. The curves of fig. 2 were computed with $z$ in the range $0.2 \leq z \leq 0.7$. These are typical HERA cuts and are intended to reduce background uncertainties due to beam gas interactions $(z>0.2)$ and DIS interactions $(z<0.7)$, they are also responsible for the cusps around $y=0.1$.

The scale $\mu$ was taken to be $p_{T} / 2$, as specified by the prescription of Ellis, Kunszt and Soper [14] who have shown that the scale $\mu$ for which the leading order calculation reproduces the less scale dependent $\mathcal{O}\left(\alpha_{s}^{3}\right)$ result is given by:

$$
\mu \approx \frac{\cosh \left(y_{1}-y_{2}\right)}{\cosh \left(0.7\left(y_{1}-y_{2}\right)\right)} \frac{p_{T}}{2} \quad \stackrel{y_{1}=y_{2}}{\longrightarrow} \quad \frac{p_{T}}{2} .
$$

This (approximate) equality of the LO and NLO cross sections for the choice of a common scale $\mu=\frac{1}{2} p_{T}$ when $y_{1}=y_{2}$ is also consistent with the results of Bödecker for two-jet direct photoproduction [15]. Varying the value of $\mu$, e.g. to $p_{T}$, does not seriously alter the curves in fig.2.

As the figure demonstrates, this is a sensitive measure since it effectively measures the ratio of the magnitude of the gluon at small $x$ to that around $x \sim 0.1$. The ratio differs by as much as $40 \%$ between the $M R S D 0^{\prime}$ and $M R S D-^{\prime}$ predictions and experiment should easily be able to distinguish between them. Note that we choose to evaluate the cross sections at $y_{1}=y_{2}$ since this maximises the sensitivity to the particular choice of gluon distribution, otherwise contributions from larger $x$ values tend to smear out the small- $x$ contribution.

Of course the predictions we have for the ratio in fig.2 does assume that one can indeed separate the direct and resolved components of the photon on an event by event basis. Recent preliminary results from HERA demonstrate that making a suitable cut on $x_{\gamma}$ (see fig.1(b)), e.g $x_{\gamma} \geq 0.75$, can provide the required clean separation [16]. Furthermore, we have checked, in $\mathrm{LO}$, that the direct cross section is almost unaltered after including the resolved component 
with $x_{\gamma} \geq 0.75$.

\section{Step 2 - the gluon in the photon}

We next turn to the resolved contribution, e.g. fig.1(b), where the two-jet cross section, to leading order, is given by

$$
\frac{d^{3} \sigma^{r e s}}{d y_{1} d y_{2} d p_{T}^{2}}=\int d z f_{\gamma / e}(z) x_{\gamma} f_{i / \gamma}\left(x_{\gamma}, \mu\right) x f_{j / p}(x, \mu) \frac{d \hat{\sigma}(i j \rightarrow k l)}{d \hat{t}}
$$

and the expression for $z$ in eqn.(2) is now the expression for the product $z x_{\gamma}$.

In the previous section, we saw that choosing $y_{1}, y_{2}$ large and positive led to small $x$ for the proton, now we see that choosing $y_{1}, y_{2}$ large and negative leads to small $z x_{\gamma}$. If we compute the average values of $x_{\gamma}$ we find that typically we are exploring the range $0.18<x_{\gamma}<0.5$ for $-2<y_{1}=y_{2}<0$.

Instead of having only two subprocesses, as in step one, we now have the full set of leading order graphs which contribute to jet cross sections in hadron-hadron scattering. We will assume that, from the analysis in step one, the small- $x$ behaviour of the partons in the proton is now sufficiently pinned down so that an analysis of the two-jet cross section can yield direct information on the partons in the photon. Again we take the scale $\mu=\frac{1}{2} p_{T}$ in the LO expressions for hard cross sections. To a large extent, the quark distributions in the photon are already constrained from fits to $F_{2}^{\gamma}$ and this is reflected in various popular parametrisations [10, 11, 17, 18] where the major differences all reside in the assumed behaviour of the gluon.

To emphasise the role of the resolved contribution we therefore shift towards more negative values of the rapidities $y_{1}$ and $y_{2}$. In fig. 3 we show predictions for the ratio of the resolved photon cross section (defined as the component with $x_{\gamma}<0.75$ ) for $-2 \leq y \leq 0$ to the direct photon two-jet cross section at $y=0$. We choose to normalise to the direct cross section at $y=0$ since this choice gives good sensitivity to the normalisation (as well as the shape) of the parton distributions in the photon, as well as ensuring that good statistics should be possible ( $y=0$ is near the peak of the direct cross section rapidity distribution). Again we integrate over $p_{T} \geq 5 \mathrm{GeV}$. It is clear from the figure that the large variations imply that the ratio is 
indeed a sensitive measure of the gluon density in the photon.

\section{Conclusions}

We have proposed that a measurement of the two-jet cross section ratio, $\sigma_{d i r}(y) / \sigma_{d i r}(-1)$, for $y_{1}=y_{2}=y$ from photoproduction at HERA will provide a sensitive test of the gluon density in the proton. The relatively large values of $y$ which are accessible enable the gluon content of the proton to be probed in the interesting small- $x$ region. Since we expect many of the experimental uncertainties involved in computing the ratio to cancel (and that any rapidity dependence of the detector acceptance can be corrected for) we expect that the construction of such a cross section ratio will be experimentally straightforward.

Already, events due to hard two-jet production at HERA have been seen clearly for $p_{T}$ up to $20 \mathrm{GeV}$ [16] - even a separation of these events into 'enriched direct $\gamma$ ' and 'enriched resolved $\gamma^{\prime}$ samples has been performed. These observations are extremely encouraging for the method we suggest since, in step one, the small- $x$ gluon in the proton is determined from the 'clean' direct photon component. Having pinned down the gluon in the proton one can then proceed to extract information on the gluon density in the photon. This is step two of our recipe in which only the resolved component of the photon contributes and where a measurement of the ratio, $\sigma_{r e s}(y) / \sigma_{\text {dir }}(y=0)$, can go a long way in discriminating between the various proposed gluon distributions of the photon.

We believe that using ratios of the cross sections in this way reduces both experimental and theoretical uncertainties and allows a relatively simple and logical procedure to provide a sensitive determination of the gluon densities in both the proton and photon.

\section{Acknowledgements}

It is a pleasure to thank Jonathan Butterworth for many helpful discussions and suggestions. 


\section{References}

[1] H1 Collaboration, I. Abt et al., DESY report DESY 93-117, Aug. 1993.

[2] ZEUS Collaboration, M. Klein et al., DESY report DESY 93-110, Aug. 1993.

[3] E.A. Kuraev, L.N. Lipatov and V.S. Fadin, Sov. Phys. JETP 45 (1977) 199;

Ya. Ya. Balitsky and L.N. Lipatov, Sov. J. Nucl. Phys. 28 (1978) 822.

[4] M. Glück, E. Reya and A. Vogt, Phys. Lett. B306 (1993) 391.

[5] A.M. Cooper-Sarkar et al., Zeit. Phys. C39 (1988) 281.

[6] A.D. Martin, C.K. Ng and W.J. Stirling, Phys. Lett. B191 (1987) 200.

[7] J. Blümlein and M. Klein, DESY report DESY 92-038 (1992);

K. Prytz, Phys. Lett. B311 (1993) 286.

[8] A.D. Martin, W.J. Stirling and R.G. Roberts, RAL preprint-93-047 (1993).

[9] CDF collaboration: contribution to the EPS Conference on High Energy Physics, Marseilles, July 1993, preprint FERMILAB-Conf-93/203-E.

[10] L.E. Gordon and J.K. Storrow, Manchester University preprint M/C.TH.92/06 (1992).

[11] M. Drees and R. Godbole, Phys. Rev. Lett. 61 (1988) 682; Phys. Rev. D39 (1989) 169.

[12] H. Baer, J. Ohnemus and J.F. Owens, Zeit. Phys. C 42 (1988) 657.

[13] A.D. Martin, W.J. Stirling and R.G. Roberts, Phys. Lett. 306B (1993) 145.

[14] S.D Ellis, Z. Kunszt and D.E. Soper, Phys. Rev. Lett. 69 (1992) 1496.

[15] D. Bödecker, Zeit. Phys. C59 (1993) 501.

[16] R. Klanner and A. de Roeck, HERA results presented at the EPS conference on high energy physics, Marseille 1993.

[17] M. Glück, E. Reya and A. Vogt, Zeit. Phys. C53 (1992) 127.

[18] H. Abramowicz, K. Charchula and A. Levy, Phys. Lett. B269 (1991) 458. 


\section{Figure Captions}

Fig. 1 (a) A direct photon contribution to the hard two-jet cross section.

(b) A resolved photon contribution to the hard two-jet cross section.

Fig. 2 The ratio $\left.\frac{d^{2} \sigma_{1}^{d i r}}{d y_{1} d y_{2}}\right|_{y_{1}=y_{2}=y} /\left.\frac{d^{2} \sigma^{d i r}}{d y_{1} d y_{2}}\right|_{y_{1}=y_{2}=-0.5}$ for three choices of proton gluon density, i.e. $M R S D 0^{\prime}, M R S D-^{\prime}$ [13] and $G R V$ [4].

Fig. 3 The ratio $\left.\frac{d^{2} \sigma^{r e s}}{d y_{1} d y_{2}}\right|_{y_{1}=y_{2}=y} /\left.\frac{d^{2} \sigma^{d i r}}{d y_{1} d y_{2}}\right|_{y_{1}=y_{2}=0}$ using (a) $M R S D-^{\prime}$ and (b) $M R S D 0^{\prime}$ for the proton parton densities. The labelled curves correspond to various choices of the photon parton densities, LAC1, LAC3 [18], GS2 [10], GRV [17] and DG [11]. Also shown are the corresponding predictions when the gluon density in the photon is switched off. 